\title{
Une mondialisation par le bas. Les daigous à Paris, des agents commerciaux intermédiaires entre producteurs français et consommateurs chinois
}

Globalization from Below. Daigous in Paris, Intermediary Sales Agents between French Producers and Chinese Consumers

\section{Simeng Wang}

\section{QpenEdition Journals}

\section{Édition électronique}

URL : http://journals.openedition.org/conflits/19664

DOI : $10.4000 /$ conflits. 19664

ISSN : $1777-5345$

\section{Éditeur :}

CCLS - Centre d'études sur les conflits lilberté et sécurité, L'Harmattan

\section{Édition imprimée}

Date de publication : 31 décembre 2017

Pagination : 107-128

ISBN : 978-2-343-14601-0

ISSN : 1157-996X

\section{Référence électronique}

Simeng Wang, «Une mondialisation par le bas. Les daigous à Paris, des agents commerciaux intermédiaires entre producteurs français et consommateurs chinois », Cultures \& Conflits [En ligne], 108 | hiver 2017, mis en ligne le 31 décembre 2019, consulté le 30 mars 2021. URL : http:// journals.openedition.org/conflits/19664; DOI : https://doi.org/10.4000/conflits.19664 


\section{Une mondialisation par le bas. Les daigous à Paris, des agents commerciaux intermédiaires entre producteurs français et consommateurs chinois}

\section{Simeng WANG}

Simeng Wang est sociologue, chargée de recherche au CNRS et membre $d u$ CERMES3 (CNRS-EHESS-INSERM-Université Paris Descartes). Elle a d'abord travaillé sur la sociogenèse et l'expression des souffrances psychiques de migrants chinois et de leurs descendants en région parisienne. Ses travaux actuels relèvent de différentes formes du transnationalisme, reliant la Chine continentale et ses diasporas. Elle a récemment publié dans les revnes Genèses (2016) et Politix (2016) et codirigé le numéro spécial "Migrations chinoises et générations 》 de la revue Hommes \& migrations (2016) ainsi que celui de la revue Participations (2017) intitulé "Participer dans le monde chinois : une jeunesse connectée ". Elle est également l'auteure de l'ouvrage Illusions et souffrances. Les migrants chinois à Paris (Paris, Éditions Rue d'Ulm, 2017).

$\mathrm{E}$ n phase de moyennisation ${ }^{1}$, la Chine se transforme progressivement en une société de consommation ${ }^{2}$. Les produits fabriqués à l'étranger, allant

1. Li P. et Zhang Y., « L'ampleur, l'identification et les attitudes sociales des classes moyennes chinoises ", Chinese Journal of Sociology (traduit du chinois par l'auteure), vol. 28, n 2, 2008, pp. 1-19; Li C., Formation of Middle Class in Comparative Perspective: Process, Influence and Socioeconomic Consequences, Beijing, Social Science Academic Press, 2009 ; Rocca J.-L., The Making of the Chinese Middle Class: Small Comfort and Great Expectations, Basingstoke and New York, Palgrave Macmillan, 2017.

2. Devenir propriétaire, voyager à l'étranger, accéder à certains produits de luxe, être attentif à son « bien-être ", défendre ses droits dans les domaines de l'environnement, de la sécurité et de la santé, sont des pratiques associées aux classes moyennes en Chine. Voir entre autres : Zheng H., La transformation sociale et la révolution des consommateurs. Les changements d'attitudes consommatrices dans les villes chinoises, Beijing, Peking University Press, 2006 ; Latham K., Thompson S., et J. A. Klein (eds.), Consuming China: Approaches to Cultural Change in Contemporary China, Londres, Routledge, 2007 ; Gerth K., As China Goes, So Goes the World: How Chinese Consumers Are Transforming Everything, New York, Hill and Wang, 2010. 
du prêt-à-porter à la nourriture, considérés comme étant authentiques et de meilleure qualité, sont fortement valorisés par les consommateurs chinois issus des classes moyennes et supérieures. Cet engouement tient en partie au manque de confiance envers les produits locaux, à la suite de scandales alimentaires ${ }^{3}$. En réponse aux besoins croissants des consommateurs chinois, on observe un renouveau des activités économiques transfrontalières. D'un côté, l'État chinois promeut le développement des partenariats et le commerce international sous diverses formes (signature de contrats de vente, création d'antennes de marketing, etc.) ${ }^{4}$; de l'autre côté, émergent de nombreuses activités économiques transfrontalières promues par des acteurs ordinaires, à titre individuel, en groupe ou en réseau. Si les actions étatiques marquent une "mondialisation par le haut ", les acteurs ordinaires participent activement quant à eux à un processus de "mondialisation par le bas 5 ». En parallèle, des activités économiques transfrontalières dans la société de consommation chinoise ont été largement marquées par la démocratisation de l'usage d'internet en Chine ${ }^{6}$. Selon le rapport annuel 7 publié par le Chinese Electronic Commerce Research Center, en 2016, les transactions d'e-commerce en Chine représentaient un total de 22970 milliards de yuan (renminbi dans le contexte chinois et en mandarin : RMB) (2938 milliards d'euros) - soit une croissance de $25,5 \%$ par rapport à l'année 2015 - et dont 5300 milliards de RMB (678 milliards d'euros) d'achats individuels ${ }^{8}$. La même année, les transactions

3. Citons par exemple les crises de sécurité alimentaire. Selon McKinsey : «after witnessing a series of food scandals over the past 5-10 years, food safety is of rising concern to Chinese consumers: $72 \%$ of consumers now worry that the food they eat is harmful to their health, up from $60 \%$ in 2012 » [ « Suite à une série de scandales alimentaires au cours de ces dix dernières années, les consommateurs chinois sont de plus en plus préoccupés par leur sécurité alimentaire : $72 \%$ se disent à présent inquiets que la nourriture soit mauvaise pour leur santé, un chiffre qui a augmenté de $60 \%$ depuis 2012 »] in Zipser D. et al., China Consumer Report: The Modernization of the Chinese Consumer, 2016, p. 9

(https://www.mckinsey.de/files/the-modernization-of-the-chinese-consumer.pdf).

4. Prenons l'exemple des commerces bilatéraux sino-français. Selon les statistiques de l'UE citées par le ministère chinois du Commerce, en 2016, le montant total atteint 46,93 milliards de dollars (équivalant à 39, 89 milliards d'euros), incluant 17,62 milliards de dollars (équivalant à 14,97 milliards d'euros) des exportations françaises en Chine. La Chine est le huitième pays d'exportation et le septième pays d'importation en France. http://countryreport.mofcom.gov.cn/record/view110209.asp?news_id=52922

5. La mondialisation par le haut porte sur l'action des États et des multinationales en vue de favoriser la libre circulation des marchandises et des capitaux. La mondialisation par le bas peut être définie comme un flux transnational de personnes et de marchandises qui impliquent des quantités relativement faibles de capital et des transactions peu institutionnalisées, informelles, parfois «semi-légales » voire "illégales ». Voir Portes A., "La mondialisation par le bas. L'émergence des communautés transnationales ", Actes de la recherche en sciences sociales, vol. 129, n 1, 1999, pp. 15-25 ; Tarrius A., La Mondialisation par le bas. Les nouveaux nomades des économies souterraines, Paris, Balland, 2002, $169 \mathrm{p}$.

6. Zhou Z., "Economic Globalization and Transformation of Consumers' Ethical Concepts in Contemporary China ", Journal of Shanghai Normal University (Philosophy E Social Sciences Edition), vol. 36, n 3 , 2007, pp. 14-20.

7. 2016 年度中国电子商务市场数据监测报告 [Rapport annuel de surveillance des données du marché du commerce électronique en Chine, 2016] http://www.100ec.cn/zt/upload_ data/16jcbg/16jcbg.pdf

8. Jusqu'en décembre 2016, 3,05 millions de Chinois étaient directement employés par le sec- 

d'euros), soit une augmentation de 28, 2 \% par rapport à l'année précédente 9 .

C'est en mettant la focale sur des acteurs ordinaires impliqués à titre individuel dans les activités économiques transfrontalières que nous étudions ici une nouvelle forme de mondialisation par le bas. Plus précisément, nous nous intéressons à un groupe d'agents commerciaux résidant à l'étranger et vendant des produits étrangers aux consommateurs chinois de Chine via des plateformes ou logiciels de transaction en ligne. Nommés daigou en mandarin, littéralement traduit comme " acheter au nom de quelqu'un d'autre », ces agents commerciaux, résidents en séjour de longue durée en France, jouent en effet un rôle d'intermédiaires entre fabricants/commerçants français et consommateurs chinois. Leur existence a modifié le modèle traditionnel du commerce international - caractérisé par une chaîne de transaction allant du producteur à l'exportateur du pays $\mathrm{A}$, puis à l'importateur, au commerçant de gros, au commerçant de détail et enfin au consommateur du pays $\mathrm{B}$ - en reliant directement le producteur du pays A et le consommateur du pays B. La suppression des étapes intermédiaires traditionnelles d'une transaction transfrontalière permet en effet au consommateur d'économiser du temps et de l'argent. Selon une enquête menée en Chine par Bain \& Company 10 , les transactions via les daigous ont augmenté de 20 milliards de RMB (3 milliards de dollars) en 2014 : en l'espace de douze mois, la consommation de produits de luxe via les daigous est passée de 55 à 75 milliards de RMB (8,8 milliards de dollars à 12 milliards de dollars). D’après un reportage réalisé par la $\mathrm{BBC}$ en octobre $2016{ }^{11}$ portant sur le phénomène en Australie, on estime qu'il y a dans ce pays environ 40000 daigous, quasi exclusivement originaires de la Chine continentale, jeunes immigrés ou étudiants.

\section{L'études des daigous : quels intérêts scientifiques, quelles approches ?}

Si les daigous ont fait l'objet d'articles ou de reportages relatifs à l'économie ou au commerce international, ils ont peu attiré l'attention des sociologues. Or, il nous semble intéressant de leur consacrer une étude sociolo-

teur de l'e-commerce et au total plus de 22,4 millions d'emplois étaient directement ou indirectement créés par ce secteur (http://finance.chinanews.com/cj/2017/05-24/8233296.shtml).

9. Par ailleurs, les experts chinois estiment que le projet de «Nouvelle route de la soie » promouvrait plus profondément l'e-commerce transfrontalier. Intitulé «One Belt and One Road» (yidai yilu), ce projet est proposé par Xi Jinping en 2013 et a pour objectif d'améliorer la connectivité entre les principaux pays d'Eurasie. L'e-commerce transfrontalier est mentionné comme un modèle innovant d'affaires et d'investissements, qui renforcera la globalisation économique de l'Eurasie (http://news.xinhuanet.com/fortune/201706/07/c_1121104111.htm).

10. Chitrakorn K., "Can China End the Illicit 'Daigou' Trade? », Business of Fashion, 5 février 2016 (https://www.businessoffashion.com/articles/global-currents/can-china-put-an-endto-the-illicit-daigou-trade).

11. Mercer P., « Shopping in Australia, while in China », BBC News, 24 octobre 2016 (http://www.bbc.co.uk/news/business-37584730). 
gique pour plusieurs raisons. D'abord, elle permet de relier une sociologie économique aux études migratoires puisque ces daigous et leurs activités économiques sont consubstantiellement liés à leur statut administratif en France et à leur projet migratoire. Analyser leurs pratiques de vente à distance, ainsi que les pratiques de maintien de la chaîne économique étendue à l'international, permet d'examiner le processus de « construction des communautés transnationales », et de revisiter les liens établis entre les réseaux, l'ethnicité et l'entreprenariat ${ }^{12}$.

Au-delà de ce premier intérêt (qui vaut pour tout type d'activités économiques transnationales), les daigous constituent un cas extraordinaire car ils exercent une activité émergente aux frontières de la légalité. En effet, d'un point de vue juridique, aucune clause n'interdit d'acheter légalement un article en France et de l'envoyer à un contact en Chine. Pourtant, de telles pratiques commerciales non seulement privent le gouvernement chinois d'importantes ressources douanières et l'État français de ressources fiscales mais nuisent également aux marchés de revente exploités en Chine pour le compte des entreprises françaises. Par ailleurs, comme nous le verrons, certains daigous, par intérêt économique, peuvent être impliqués dans la commercialisation des contrefaçons. Ainsi, développé à partir d'une économie informelle ${ }^{13}$, le secteur de daigous fait l'objet d'une tentative de régulation par le gouvernement chinois, qui relève d'une mondialisation par le haut. À ce titre, l'étude des daigous peut mettre en lumière une tension entre les deux types de mondialisation, entre d'un côté des tactiques développées par les individus pour contourner les sanctions douanières, et de l'autre une série de régulations étatiques visant à proscrire ces activités économiques.

Enfin, une telle étude peut combler un manque de données statistiques sur ces activités économiques quantitativement insaisissables car exercées aux marges de la légalité. Notre analyse qualitative vise à produire quelques connaissances sur les profils et trajectoires sociales des daigous - comment chaque daigou choisit d'exercer cette activité - ainsi que sur les conditions sociales aux niveaux méso et macro permettant l'émergence d'une telle filière. Au niveau mésosocial, il s'agit de comprendre les relations que ces daigous entretiennent avec des commerçants chinois dits "classiques », majoritairement originaires de la région de Wenzhou, immigrés depuis plus d'un siècle en France ${ }^{14}$ et positionnés dans les commerces d'import-export sino-français de longue date, bien avant l'ère numérique et l'arrivée de l'e-commerce. Autrement dit, nous cherchons à savoir s'il y a lutte et/ou collaboration entre

12. Portes A., "La mondialisation par le bas. L'émergence des communautés transnationales », op. cit. ; Portes A. (ed.), The Economic Sociology of Immigration: Essays on Networks, Ethnicity, and Entrepreneurship, New York, Russell Sage Foundation, 1995.

13. Lautier B., L'Économie informelle dans le tiers monde, Paris, La Découverte, 2004 ; Peraldi M. (dir.), Cabas et containers : Activités marchandes informelles et réseaux migrants transfrontaliers, Paris, Maisonneuve et Larose, 2001 ; Tarrius A., op. cit. 
les daigous et les commerçants « classiques » contraints d'adapter leurs pratiques professionnelles aux nouveaux enjeux économiques. Qui commercialise quoi et quid de la division du travail lorsqu'un partenariat se crée entre ces deux types de commerçants ? Ces questions permettent de cerner les niches économiques dans lesquelles émerge la filière daigou et nous conduisent à réfléchir sur la nature de ces activités : pourraient-elles être qualifiées de «métier», de «profession»?

Au niveau macrosocial, nous tentons d'analyser dans quelle mesure l'émergence des activités daigou répondrait à la croissance de la société de consommation chinoise, à l'engouement pour les produits fabriqués à l'étranger (garants d'authenticité et de qualité), à une recherche de prix relativement " raisonnables» (moins élevés que ceux affichés en Chine) et, plus profondément, à un manque de confiance envers les produits locaux. Ceci s'observe davantage pour les denrées alimentaires - en raison des scandales alimentaires chinois 15 - que pour les produits de luxe. Cette évolution des formes de transaction commerciale entre la Chine et la France est également traversée par des tensions entre les voies « par le haut » et « par le bas » de la mondialisation.

\section{De l'importance de prendre en compte «le facteur temps »}

Si l'articulation des échelles analytiques (micro, méso et macro) est nécessaire pour appréhender la filière daigou, la prise en compte de la dimension temporelle est tout aussi cruciale. Lorsque nous étudions à l'échelle individuelle le profil de ceux qui prennent part aux activités daigou, nous nous apercevons qu'il s'agit majoritairement de femmes, à des cycles de vie spécifiques, et que l'approche en termes de «trajectoire sociale » s'avère féconde. Pour les analyses d'échelle méso et macrosociales, c'est l'approche historicisée qui apparaît comme la plus pertinente, et ce à trois égards.

En premier lieu, en raison de la place de l'économie chinoise dans la chaîne de production mondiale. Il y a quelques siècles, d'« anciens » commerçants

14. Leur flux migratoire remonte au début du XXe siècle avant de connaître un rebond à partir des années 1980. Majoritairement issus du milieu rural avant leur émigration et disposant d'un capital culturel relativement faible, ils émigrent principalement à des fins économiques et avec l'objectif d'emmener l'ensemble de la famille. Bénéficiant souvent d'un réseau social fondé sur la parenté élargie et la relation entre compatriotes, les Wenzhous commencent par travailler au sein d'une structure économique appartenant à un membre de la «communauté » et finissent si possible par ouvrir leur propre commerce. Sur l'histoire des Wenzhous en France, voir entre autres, Live Y.-S, Guillon M. et Ma Mung E., « Les Chinois de Paris depuis le début du siècle. Présence urbaine et activités économiques ", Revue européenne de migrations internationales, vol. 8, n³, 1992, pp. 155-173; Ma Mung E., « Le commerce ethnique des immigrés chinois en France ", Approches Asie, n 17, 2000, pp. 55-64 ; Poisson V., " Des réseaux transnationaux : le cas des Chinois du Zhejiang ", Outre-Terre, vol. 17, n 4, 2006, pp. $421-430$.

15. Voir entre autres Klein J. A., «Everyday Approaches to Food Safety in Kunming », The China Quarterly, vol. 214, 2013, pp. 376-393. 
wenzhous ont entamé leur carrière en faisant du commerce de produits intensifs en main-d'œuvre ciblant le marché français, fabriqués par leurs compatriotes en France ou exportés de Chine, et notamment de la région de Yiwu ${ }^{16}$. C'était l'époque où la Chine, considérée comme l'usine du monde, offrait une main-d'œuvre très bon marché. De ce fait, ces commerçants wenzhous se sont moins spécialisés dans les flux commerciaux de produits français vendus en Chine, et encore moins dans l'e-commerce. Au fil du temps, le développement économique de la Chine a fortement accru le pouvoir d'achat des classes moyennes et supérieures chinoises (voir les statistiques du ministère du Commerce susmentionnées). La structure et le sens des flux commerciaux entre la Chine et la France ont connu de profondes reconfigurations.

Ensuite, l'approche historicisée nous aide à mieux comprendre l'usage croissant des nouveaux médias dans le domaine de la transaction économique à distance. Les plateformes de réseaux sociaux comme Wechat (créée en 2011) offrent un support de communication. Les transactions monétaires sont faciles à opérer, instantanées et sans partage direct de données bancaires entre commerçant et client, grâce aux applications de paiement telles qu'Alipay (créée en 2004) et Wechat (dans la fonction " portefeuille»). Avec ces supports technologiques, le nombre de transactions via daigous a atteint 70 milliards de RMB (8, 9 milliards d'euros) en 2013 et dépassé 150 milliards de RMB (19 milliards d'euros) ${ }^{17}$ en 2014. Selon un sondage effectué par Exane BNP Paribas auprès de 5500 e-consommateurs chinois résidant dans quarante-huit villes, $35 \%$ des clients achètent des produits de luxe via les daigous, contre $45 \%$ en ligne via les sites chinois, $13 \%$ via les sites étrangers, et $7 \%$ seulement commandent directement via le site des marques de luxe 18 .

Enfin, au fil du temps, la composition démographique et sociologique de la population chinoise en France a également connu de profondes évolutions. Premièrement, depuis le début des années 2000, on peut observer l'arrivée massive de jeunes Chinois qui souhaitent poursuivre des études supérieures, qui décident de résider en France une fois celles-ci achevées et deviennent ainsi des migrants qualifiés. En 2011, la Chine devient le deuxième pays d'origine des étudiants étrangers présents en France, après le Maroc ${ }^{19}$. Selon un reportage paru le 5 août 2013 dans France diplomatie, en l'espace de dix ans, le nombre d'étudiants chinois en France a été multiplié par dix. Lors d'un entretien en novembre 2014, M. Ma, le ministre-conseiller (service de l'éducation) de

16. Guiheux G., « Travailleurs migrants du prêt-à-porter en Chine. Flexibilités et opportunités ", Revue européenne des migrations internationales, vol. 28, n 4, 2012, pp. 27-42.

17. http://www.sohu.com/a/27762820_216836

18. Solca L., « Digital China Leaving Ostrich Brands Behind », Business of Fashion, 16 avril 2015 (https://www.businessoffashion.com/articles/opinion/digital-china-leaving-ostrich-brandsbehind).

19. En 2010-2011, la France a accueilli 284659 étudiants étrangers. Les étudiants chinois représentent $12 \%$ du total. Parmi les doctorants étrangers, $41 \%$ viennent de la Chine. 
l'Ambassade de Chine en France, nous a indiqué qu'à cette date, 40000 étudiants chinois vivaient dans l'Hexagone en vue d'obtenir un diplôme français (allant du Bac au doctorat). La moitié d'entre eux résidait en Île-de-France. Les trois principales disciplines étudiées sont le commerce (la plus choisie), les sciences dures (théoriques et appliquées, incluant les sciences de l'ingénieur) et les sciences sociales et humaines. Les lieux de scolarité privilégiés sont les grandes écoles (d'ingénieurs, de commerce ou de sciences sociales) et les universités ${ }^{20}$. La poursuite des études est devenue le premier motif migratoire des Chinois en France, ce qui nous amène à qualifier cette arrivée massive de jeunes chinois qualifiés de quatrième vague migratoire des Chinois vers la France ${ }^{21}$. Ce sont justement parmi ces primo-arrivants chinois qualifiés - originaires de diverses régions de Chine, mais rarement de la région de Wenzhou - que nous trouvons les futurs daigous. Nous verrons en détails leur trajectoire sociale.

En ce qui concerne les commerçants wenzhous, c'est au moment charnière de la reprise de l'entreprise du père par le fils que s'opère un renouvellement des activités économiques. Pour la plupart nés en France, les enfants basculent d'un modèle traditionnel de commerce transfrontalier à un modèle d'ecommerce. Comme le dit Alexandre, fils de M. Liu, grossiste de chaussures installé à Aubervilliers :

« La génération de mon père tire son intérêt de la quantité de marchandises vendue, avec une main d'œuvre extrêmement peu chère en Chine à leur époque. Mais les temps changent. Aujourd'hui, le plus grand marché du monde est en Chine. Les consommateurs chinois sont de plus en plus connectés et achètent pour la qualité [du produit]. Donc notre génération doit impérativement songer à une transition professionnelle et se positionner par rapport aux nouveaux besoins de la clientèle ! " (Entretien mené à Aubervilliers, en septembre 2014)

Après la reprise, Alexandre se positionne en effet en tant qu'e-commerçant et collabore occasionnellement avec des daigous.

20. Wang S., "Des "soucis matrimoniaux" dans le contexte transnational : le cas des migrants et des migrantes chinois qualifiés à Paris ", Migrations Société, vol. 27, n 157, 2015, pp. 149-166.

21. Wang S., Illusions et souffrances. Les migrants chinois à Paris, Paris, Rue d'Ulm, 2017. Sur les vagues précédentes de l'immigration chinoise en France, voir par exemple Ma Mung E., « La diaspora chinoise en France", in Poinsot M. et Weber S. (dir.), Migrations et mutations de la société française, l'état des savoirs, Paris, La Découverte, 2014, pp. 121-129. 
Une enquête qualitative auprès de daigous, de leurs partenaires commerciaux et de leurs clients

Pour approcher empiriquement le global ${ }^{22}$, nous nous appuyons sur des matériaux de première main collectés depuis 2010 en étudiant des trajectoires et expériences migratoires de Chinois à Paris. Au fil du temps, nous avons mené une trentaine de sessions d'observation participante (plus de 100 heures au total) dans les lieux de vente (galeries, boutiques de marques et pharmacies) à Paris, où les daigous font des courses pour satisfaire les demandes et commandes de leurs clients, parfois connectés en ligne. Par ailleurs, nous avons mené des entretiens semi-directifs avec une vingtaine de daigous - dont la moitié travaillent exclusivement en tant que daigou et l'autre moitié ont un emploi salarié principal et considèrent l'activité de daigou comme un emploi à temps partiel. Nous avons également rencontré depuis 2010 une dizaine de daigous étudiants, avec qui nous avons eu des discussions informelles, conduites au cours d'activités amicales organisées par des associations chinoises et franco-chinoises (comme celles d'anciens étudiants d'universités chinoises en France, et d'autres de loisirs).

En sus des entretiens menés auprès de daigous, nous avons mené une dizaine d'entretiens avec différents acteurs-clés de ce type de commerce transfrontalier et notamment avec des vendeurs de produits de luxe, cosmétiques et alimentaires en France. Nous avons ensuite rencontré trois types de partenaires commerciaux des daigous : des commerçants wenzhous qui recrutent des daigous pour évaluer la situation de l'e-commerce en Chine ou pour commercialiser des produits de contrefaçons; des migrants chinois de milieux populaires qui sont en quelque sorte "embauchés » par des daigous pour aller faire la queue devant les boutiques de produits de luxe à leur place, ou leur faire la cuisine; et des Chinois qualifiés exerçant des professions libérales dans le domaine du tourisme, qui aident les daigous à trouver des passeports de touristes chinois afin de pouvoir détaxer leurs achats ${ }^{23}$.

En parallèle, nous avons mené une dizaine d'entretiens complémentaires en Chine, auprès de consommateurs chinois ayant déjà procédé à des achats via des daigous en France. Ils nous ont permis de mieux comprendre leurs attentes de consommateurs quant aux produits fabriqués dans l'hexagone, et de recueillir leurs avis sur les activités des daigous.

22. Burawoy M. et al., Global Ethnography: Forces, Connections, and Imaginations in a Postmodern World, Berkeley, University of California Press, 2000 ; Rossi I. (ed.), Frontiers of Globalization Research. Theoretical and Methodological Approaches, New York, Springer, 2017 ; Siméant J. (dir.), Guide de l'enquête globale en sciences sociales, Paris, CNRS Edition, 2015.

23. Pour les résidents chinois âgés de 16 ans ou plus à la date des achats, de passage en France pour moins de six mois, la TVA peut être déduite du prix des marchandises qu'ils achètent en France. Voir http://www.douane.gouv.fr/articles/a10806-detaxe-conditions-d-eligibilite. 
Enfin, quelques entretiens réalisés avec des responsables politiques chinois - notamment au sein du bureau du conseiller économique et commercial et la section de l'éducation de l'Ambassade de Chine en France - nous ont offert une vision générale sur l'évolution des politiques d'accompagnement mises en œuvre pour le développement de l'e-commerce transfrontalier et sur la volonté gouvernementale du contrôle de la filière daigou.

\section{Une filière «commandée » par le marché : enjeux de confiance et de taxes}

Selon les statistiques nationales, en 2013, les deux groupes de consommateurs les plus exposés à l'e-commerce étaient les Chinois âgés de 25-30 ans (39,7 \% des transactions de produits importés) et de 31-35 ans (25,3\%). Les trois principales raisons qui motivent l'achat de produits importés sont : l'inaccessibilité du produit en Chine (guonei maibudao, $52 \%$ ); la garantie de qualité du produit (pinzhi baozheng, 50,6 \%) ; le prix bon marché (jiage pia$n y i, 50,3 \%$ ). Les autres raisons évoquées sont la recommandation des amis, la variété des modèles et la qualité du service. Avec la démocratisation des smartphones et le développement des applications d'achat, les partages d'expériences de shopping en ligne deviennent une pratique courante. Acheter des produits importés de l'étranger permet d'afficher non seulement son pouvoir d'achat ${ }^{24}$ dans son cercle de sociabilité, mais aussi la personnalité et les valeurs que l'on souhaite mettre en avant : curiosité, esprit de découverte ou d'aventure, perfection de la qualité de vie, etc.

Jeunes, qualifiés, ayant des revenus élevés, et principalement de sexe féminin, ces consommateurs sont notamment à la recherche de trois catégories de produits importés : les produits de luxe (textile haute couture, montres, sacs de marque), les produits cosmétiques et les produits alimentaires, particulièrement à destination des bébés ${ }^{25}$. Les clients que nous avons interrogés expliquent avoir recours aux daigous car ils sont une garantie de la qualité du produit. Une perte de confiance envers les produits fabriqués en Chine pousse ces consommateurs à recourir à ce mode d'achat. Le fait d'avoir un interlocuteur, au moment de l'achat, susceptible de présenter les produits voire de fournir quelques conseils, de manière quasi instantanée, est pour ces clients gage d'un service de qualité car « personnalisé ». La confiance accordée à un agent daigou contribue largement à la popularité de ce mode d'achat. Le discours de madame Li est à ce titre révélateur :

« En Chine, on est submergé par des scandales de faux produits, allant des peintures toxiques à l'huile recyclée. L'histoire par exem-

24. Certains consommateurs en profitent pour exhiber leur richesse. Sur ce type de pratique, voir entre autres Jiang J., "Exhiber la richesse en ligne : la pauvreté spirituelle et la perte de valeur ", Modern Communication, $\mathrm{n}^{\circ}$ 2, 2013, pp. 15-19.

25. Avec la fin de la politique de l'enfant unique en 2015, la Chine pourrait connaître, dans les années qui viennent, un baby-boom. 
ple de la farce de la brioche chinoise fabriquée à partir de papiers journaux est hallucinante. Chez nous, la plupart des produits alimentaires viennent de l'étranger. Depuis ma grossesse, nous avons décidé de changer nos habitudes de consommation, pour la santé de notre enfant. À ce moment-là, j'ai fait la connaissance de Mei, une amie d'amie, qui venait de commencer ses activités de daigou à Paris, spécialisée dans des produits destinés aux mères et aux enfants. Nous avons vite sympathisé et aujourd'hui j'achète encore chez elle, et aussi chez d'autres daigous par son intermédiaire. » (Madame Li, habitant à Beijing, juriste, 35 ans, mère d'un enfant de deux ans ; entretien mené en novembre 2015 à Beijing)

L'activité de daigou est ici avant tout présentée comme une des réponses au manque de confiance envers les produits locaux et comme un service personnalisé. Par ailleurs, la dimension économique, autrement dit le coût relativement bas des produits étrangers, motive grandement les consommateurs à recourir à ce mode d'achat qui permet de contourner certaines taxes. En effet, la douane chinoise distingue deux types d'entrée de produits importés en territoire chinois : les «marchandises » (buown) et les «objets» (wupin). Cette distinction s'appuie sur deux critères : la nature « commercialisée » (ou non) du produit, ainsi que la quantité de produits envoyés. Les taxes d'importation appliquées varient en fonction de ces critères. Les produits envoyés par les daigous sont considérés comme des « objets » de consommation à titre personnel et en quantité raisonnable. Intitulée «personal postal articles tax» (xingyoushui), la taxe à payer varie de $15 \%$ à $60 \% 26$ et l'exonération est fixée à 50 RMB. Autrement dit, lorsque le montant de la taxe est inférieur à $50 \mathrm{RMB}$, elle est exemptée. Alors que si un même produit est catégorisé comme « marchandise », trois types de taxe sont obligatoires : la taxe de douane (10\% dans le meilleur des cas : en provenance de most-favoured-nation-zuibuiguo), la taxe sur la valeur ajoutée (usuellement $17 \%$ ) et la taxe de consommation (entre $0 \%$ et $30 \%$ ), soit un total de $27 \%$ de taxes dans le meilleur des cas.

Le différentiel de taxe entre ces deux catégories est plus ou moins important selon le produit concerné, ce qui explique en partie les types de produits que les daigous rencontrés choisissent de commercialiser : les produits de luxe, cosmétiques et alimentaires (comme le lait en poudre) sont ceux qui présentent le plus grand écart de prix avec le marché chinois. Plusieurs enquêtés soutiennent qu'ils ont eu l'idée de devenir daigou après avoir rendu de nombreux services à leur entourage, qui commandait tels ou tels produits français. C'est notamment le cas de Leiqi, une femme de 30 ans :

26. Il s'agit des nouvelles politiques de taxe, mises en place le 8 avril 2016. Avant cette date, la taxe "personal postal articles tax» (xingyoushui) variait de 10 à $50 \%$. Le changement des taux, parmi d'autres mesures gouvernementales, marque la volonté de l'État chinois de réguler ces activités de daigou. 
«Autour de 2010-2011, j'étais en master marketing. Au moins trois ou quatre fois par mois, des proches et des amis de Chine me demandaient de leur acheter un truc en France : portefeuilles, sacs à main, lotions de beauté... Au départ, c'était à la demande de mes parents, mes tantes ou leurs très bons amis. J'ai donc aidé sans hésitation. Mais au fil du temps, cela devenait ingérable parce qu'avec les réseaux de sociabilité de mes proches, certaines nouvelles commandes sont arrivées de la part de gens que je ne connaissais pas, que je ne me sentais pas obligée d'aider. L'idée de leur demander une commission est venue comme ça. Et en 2013, j’ai officialisé ce statut de daigou vis-à-vis de mes clients chinois... » (Entretien mené à Paris, en mars 2015)

\section{Devenir daigous : trajectoires multiples et profils sociaux relativement homogènes}

Actrice d'une mondialisation par le bas, Leiqi a décidé de devenir daigon en 2013, deux ans après son recrutement dans une agence de publicité, où elle travaille encore en CDI lors de notre dernier entretien. Elle a fait ce choix lorsque le moment propice s'est présenté : d'un côté, elle a pu changer de titre de séjour (d' «étudiant » à « salarié ») et de l'autre côté, elle commençait à mâ̂triser son travail salarié, désormais « routinier » et « peu prenant en termes de temps et d'énergie». Ces conditions de travail lui ont permis de développer ses activités de daigou en parallèle. Son profil professionnel est représentatif d'une majorité de nos enquêtés, parmi lesquels nous trouvons des salariés de différents secteurs (ingénieurs, informaticiens, vendeurs ${ }^{27}$, etc.), qui justifient leur choix de devenir daigous avant tout par intérêt financier.

«Je suis un jeune papa [son enfant, né à Paris, a 1 an]. Cette activité me permet d'acheter le lait en poudre de mon fils! Ça va bientôt faire sept ans que je suis dans cette entreprise, c'est mon premier boulot. Il y a peu de possibilités de promotion et le salaire stagne. Avec sa grossesse, ma femme avait un risque élevé de fausse couche et elle a donc arrêté de travailler. Du coup c'était à moi seul de nourrir toute la famille. Vu que je ne connaissais absolument rien à la mode, je me suis lancé dans l'alimentation, le vin et les alicaments (baojianpin). C'était très prenant comme boulot au départ, mais c'est assez rémunérateur. Nos revenus mensuels ont pu doubler. » (Qing, 34 ans, ingénieur ; entretien mené en 2015)

En fonction du temps investi dans ces activités, du type de produits commercialisés et de la commission (entre 5 et $15 \%$ ), les daigous que nous avons

27. Ce sont des vendeurs salariés dans les boutiques de produits de luxe ou de produits cosmétiques, qui décident de faire daigon en parallèle de leur activité professionnelle principale. 
rencontrés gagnent entre 1200 et 5000 euros par mois. La nature rémunératrice de cette activité attire également un autre profil d'enquêtés que nous avons rencontrés : des travailleurs indépendants dans les domaines des arts et cultures : acteurs, artistes, bloggeurs, etc. Faute de revenus réguliers, ces personnes comparent leur activité de daigon avec les petits boulots de maind'œuvre.

«Il y a dix ans, quand je suis venue en France pour ma licence, j'ai fait des petits boulots : plongeur au restau, serveur dans un café, j'ai fait la cueillette des pommes en Normandie... À mes yeux, daigou c'est un boulot du même ordre. Pas besoin de diplôme. Tous les jeunes, même des moins jeunes, à partir du moment où ils ont du temps, un smartphone, un compte Wechat et un peu de fonds de départ, peuvent le faire. Si on veut bien le faire, ça prend du temps et de l'énergie mais l'avantage est que ça rapporte bien mieux que d'autres boulots. À vrai dire, merci à l'économie chinoise et aux nombreux consommateurs de nous donner cette opportunité de nous nourrir [rire] ! » (Aiqiu, chanteuse, 28 ans, entretien mené en mai 2015 à Paris)

Un troisième profil de daigous est celui des jeunes femmes devenues mères et/ou futures mères. Toutes en rupture professionnelle en raison de leur grossesse et/ou du bas âge de leur enfant, elles sont amenées à fréquenter des forums de soins néonataux et périnataux en mandarin, et découvrant ainsi la forte demande en matière de produits maternels et infantiles - lait en poudre, poussettes, couches, jouets, entre autres -, elles décident de devenir daigous dans ce secteur. Examinons le cas de Yue, 36 ans, ingénieure, ancienne salariée d'une entreprise française. Mère de deux enfants, elle a démissionné et travaille actuellement à temps plein en tant que daigou.

«C’est une coïncidence que je me sois lancée dans cette activité. En surfant dans les forums dédiés aux mamans chinoises il y a quatre ans, je me suis rendue compte que ces mères accordent énormément d'importance aux produits alimentaires de leurs bébés, à la suite des crises du lait frelaté en 2004 et 2008 28. Certaines, après avoir su que j'étais en France, me demandaient de leur envoyer des boîtes de lait en poudre par la poste. Avec l'effet des réseaux sociaux, il y avait de plus en plus de demandes. Je me suis donc dit : "pourquoi ne pas travailler dans ce secteur ?” Il était encore peu développé par rapport à celui des produits de luxe. En plus, il s'agit de consommations couvrant une relativement longue période, non périssable, dans le

28. Il s'agit de la contamination du lait par la mélamine en 2008. Certains lots de lait de consommation courante et de lait infantile produits en Chine ont contenu pendant dix mois de la mélamine, toxique, afin de les faire paraître plus riches en protéines. Ont été reportés des dizaines de milliers de cas de malades. 
sens où un bébé grandit, on va devoir lui changer de lait, de jouet, et il y a donc toujours un besoin. Et les parents chinois, ils n’hésitent pas à dépenser pour leurs enfants [...]. » (Entretien mené en avril 2013 à Paris)

Depuis 2016, le chiffre d'affaire de Yue a connu un nouvel essor depuis la fin de la politique de l'enfant unique en décembre 2015 car certaines de ses clientes entament une seconde grossesse et l'enfant occupe toujours une place centrale dans une famille urbaine de classe moyenne ou supérieure. Le budget alloué à l'enfant au sein d'un foyer demeure relativement élevé.

Enfin, un dernier profil de daigous est celui des étudiants nés dans les années 1980 et 1990, dont majoritairement des femmes. Pour ces connaisseurs de la mode et passionnés de shopping, devenir daigou permet d'arrondir les fins de mois. Encore en phase d'études, ils sont moins impliqués dans la commercialisation des aliments ou des produits maternels et infantiles - secteur exclusivement réservé à des jeunes mères comme Yue - et se spécialisent plutôt dans les produits de luxe et cosmétiques (soin et maquillage).

Il est difficile d'estimer le nombre de daigous chinois en France. Certains journalistes français parlent de « centaines » de daigous exerçant à Paris ${ }^{29}$. Les éléments collectés lors de nos entretiens, qui croisent les points de vue de différents acteurs, offrent quelques informations complémentaires à ce sujet.

"Parmi mes connaissances chinoises en Europe, sept sur dix sont daigous. » (Hua F., 29 ans ; entretien mené en février 2015 à Paris)

«En moyenne, j'accueille environ cent clients chinois [par jour], dont deux tiers sont des daigous, dont beaucoup d'étudiantes; et le reste sont des touristes chinois [...] On voit aussi les daigous venant d'autres pays, notamment du Japon et de la Corée. Mais je dirais qu'environ $85 \%$ de daigous venus chez nous sont des Chinois. » (Elsa, Chinoise, vendeuse chez Hermès aux Galeries Lafayette ${ }^{30}, 32$ ans ; entretien mené en décembre 2014 à Paris)

« À ma connaissance, il existe des milliers de jeunes Chinois, souvent des femmes, faisant daigous. Ils ne sont pas les concurrents de mon entreprise [...]. » (M. Liu, originaire de Wenzhou, présidentdirecteur général d'une entreprise d'e-commerce transfrontalier à Paris, 41 ans ; entretien mené en avril 2015 à Paris)

29. Forta V., "À Paris, les “Daigou”, assistants shopping 2.0 des Chinois ", Asialyst, 2 août 2017 (https://asialyst.com/fr/2017/08/02/paris-daigou-assistants-shopping-2-0-chinois/)

30. Selon Elsa, en l'espace de trois ans (2011-2014), toutes les marques de la galerie ont embauché au moins deux vendeurs sinophones, pour accueillir les clients chinois dont le nombre est en forte croissance. 


\section{Une économie informelle ramifiée et hiérarchisée}

Afin de maximiser les marges bénéficiaires, les daigous tentent de saisir toute opportunité de faire baisser du coût lors de leur achat : soldes, ventes privées pour les clients VIP, détaxe de la TVA, etc. Par ailleurs, l'exclusivité des produits devient un enjeu crucial qui leur permet d'être compétitifs par rapport à la concurrence. L'exemple donné par Jun l'illustre :

"Je ne fais que les produits de maquillage. Prenons les rouges à lèvres. Certains numéros [couleurs] de certaines marques, les plus vendus et trop à la mode, sont en rupture de stock en Chine. Du coup, dès que je vois des produits disponibles ici, même si sur le moment je n'ai pas de demande, je prends tout, j'achète tout le stock $\mathrm{du}$ magasin. Parce que je sais qu'ils seront vendus à des Chinois [consommateurs], et au pire, je les vends à mes collègues [d'autres daigous comme elle]. » (Jun, 25 ans, étudiante ; entretien mené en janvier 2016 à Paris)

Ce type de pratique qui consiste à monopoliser le marché local n'est pas spécifique au secteur cosmétique et crée de nouvelles étapes de transaction au sein du même groupe de daigous. Une autre daigou spécialisée dans le lait en poudre nous raconte avoir vidé une fois le stock d'un magasin en achetant trois cents boîtes d'un coup puis les avoir revendues à ses homologues daigous cinquante centimes d'euros supplémentaires par boite. Dans le secteur de la haute couture, l'exclusivité du produit devient parfois l'élément le plus décisif d'une transaction. Certains daigous moins expérimentés sont parfois « employés » par ceux dotés de réseaux de clientèle pour aller chercher spécifiquement les modèles commandés par les clients. Comme le dit Qingqing (22 ans, étudiante) : "Je démarre tout juste. Jeune et en pleine forme, ça ne me dérange pas de faire de la route et passer mon temps à chercher des modèles. Ces derniers temps, je passe mes journées à la Vallée village et collabore avec différents grands daigous [ceux ayant des fichiers clientèle]. En me connectant en ligne, tous nos échanges sont instantanés ».

Au-delà des ressources sociales - en particulier le développement et le maintien du réseau de clientèle -, les daigous, pour mieux gérer leurs activités, doivent être dotés d'autres types de ressources. Savoir analyser le marché chinois en est une.

\section{"Maîtriser le marché chinois, c'est aussi mâ̂triser les politiques chinoises "}

Ting, 38 ans, chef d'entreprise de formation en œnologie, était un daigou du vin (2010-2014). Il nous explique son changement professionnel en ces termes : 
«En Chine, le commerce ne peut pas se faire si l'on n’étudie pas la politique! Pas uniquement les politiques économiques, mais également la vie politique chinoise. Certains daigous du vin se sont fait avoir par les politiques anti-corruption de Xi Jinping car ils stockaient trop de vin de grandes marques. Avant on arrivait à les vendre parce qu'une bouteille qui coûtait plus de mille euros faisait un beau cadeau, surtout dans le milieu politique. Quant à moi, heureusement j'avais pu sentir ce tournant des politiques et me suis vite débrouillé pour vider mes stocks de Lafitte. » (Entretien mené en mars 2015 à Paris)

Ting parle du début de la mise en œuvre des politiques anti-corruption en 2012, qui a résolument modifié la demande en vin rouge sur le marché chinois ${ }^{31}$. En effet, les vins de grandes marques sont moins recherchés et la moyennisation de la société chinoise continue à faire croître la demande en vin de moyenne gamme, dont le prix en France varie entre 8 et 50 euros. C'est ainsi grâce à la prévision des futures tendances du marché chinois que Ting a ajusté ses stocks et réorienté sa carrière professionnelle : étant donnée la forte croissance des commandes de vin rouge de la part des classes moyennes chinoises, il s'est lancé dans la formation en œnologie en langue chinoise.

Le marché des produits de luxe, comme celui du vin, a également connu les influences majeures des politiques anti-corruption : en 2014, les transactions liées aux achats d'habits pour hommes et de montres de luxe ont largement chuté par rapport à la croissance de la demande en vêtements et de chaussures pour femmes. Plusieurs enquêtés daigous ont par conséquent réajusté leur spécialisation.

\section{Combiner le daigou des produits avec la prestation des connaissances et ser- vices}

Comme Ting, certains daigous (souvent celles et ceux ayant un niveau d'études de master 2) associent voire combinent l'e-commercialisation des produits avec une prestation de service et de connaissance, qui leur permettent, selon leurs propres termes, de «fidéliser une clientèle à travers une expertise ».

Yue, daigou spécialisée dans les produits maternels et infantiles propose en parallèle de ses ventes une formation en ligne payante en soins de la petite enfance à destination des parents chinois. L’idée lui est venue à la suite d'une

31. Selon les statistiques de la douane chinoise, l'import de Bordeaux rouge en Chine s'élevait à 43,6 millions de litres en 2011 (avec une croissance de $91 \%$ par rapport à l'année précédente) ; à 53,8 millions de litres en 2012 (croissance de $23 \%$ ) ; à 45,2 millions de litres en 2013 (diminution de $16 \%$ ) ; et dans la première moitié de l'année 2014 à 32,5 millions de litres (baisse de $28 \%$ ). 
affaire de commotion cérébrale (naozhendang) d'un bébé causée par les mouvements de son père [qui essayait de calmer l'enfant en le berçant]. Yue fait intervenir une assistante maternelle de son quartier et assure elle-même la traduction en mandarin.

Au fil du temps, le corps professionnel des daigous se ramifie et une hiérarchie professionnelle se crée. Les mieux dotés en réseaux de clientèle deviennent en quelque sorte les «patrons » d'autres daigous. Les mieux pourvus en ressources scolaires, culturelles et linguistiques multiplient leurs activités. Ils ne se contentent plus de commercialiser des produits mais proposent des services et prescrivent des connaissances.

Ces évolutions intra-professionnelles observées au sein du corps daigon méritent d'être analysées en parallèle aux conséquences interprofessionnelles entraînées par cette activité. Des nouvelles activités économiques se développent en amont et en aval des daigous.

\section{Tout devient commercialisable ? Les activités économiques dérivées des daigous}

Résidents en séjour de longue durée en France, les daigous ne remplissent pas les conditions nécessaires pour bénéficier de la détaxe. Ils sont ainsi à la recherche de passeports de touristes chinois leur permettant de baisser le coût d'acquisition des produits grâce à la détaxe à laquelle ces derniers ont droit. D'autant plus que certaines marques (notamment des produits de luxe), familières du mode d'achat daigou, limitent le nombre d'achats par pièce d'identité durant un temps déterminé ${ }^{32}$. Pour être mis en contact avec des touristes chinois, les daigous sont amenés à collaborer avec des guides touristiques. Jian, 28 ans, guide, nous raconte ses partenariats avec des daigous.

« Notre bus [de tourisme] se gare toujours à côté des galeries Lafayette. Quand mes clients sont partis faire du shopping, c'est souvent le moment où les daigous viennent me chercher. Ils me disent de combien de passeports ils ont besoin ce jour-là. Et de mon côté, je vais regarder parmi les premiers rentrés au bus qui a acheté quoi, en gros, qui serait partant pour prêter sa pièce d'identité. Ensuite je leur demande en privé et s'ils sont d'accord je file leur passeport au daigou. C'est pendant le temps d'attente [pour que tous les touristes soient revenus] que le daigou finalise son paiement avec les passeports de mes clients. Normalement, [l'usage d']un pas-

32. Certaines marques mettent les daigous sur liste noire en raison d'achats trop fréquents. Elles considèrent que ce mode d'achat nuit au profit de vente sur le marché chinois et augmente le risque de contrefaçons - nous le développerons plus loin. Dans l'idée de contrecarrer le mode d'achat daigou, certaines marques (comme Chanel) baissent leurs prix en Chine, voire augmentent en même temps leur prix en Europe. 
seport coûte trente euros au daigou et j'en récupère dix. » (Entretien mené à Paris en janvier 2014)

Le partenariat créé entre guides touristiques et daigous est similaire à celui établi entre ces derniers et les vendeurs de certaines marques. Même si la politique de la boutique leur interdit de collaborer avec les daigous, certains vendeurs, en vue d'augmenter leur chiffre de vente, maintiennent de «bons contacts " avec eux, en leur proposant des nouveautés, en les invitant aux ventes privées, ou en les prenant en photos lors d'essayages - souvent demandés par les clients chinois des daigous quand il s'agit de produits de luxe contre une rémunération (entre dix et vingt euros par essai).

Par ailleurs, certaines activités économiques émergent au sein de la population chinoise à Paris, autour du mode d'achat daigou. L'une d'elle consiste à " accompagner l'achat » : il s'agit de payer quelqu'un pour qu'il/elle participe à l'acquisition d'un produit en vue de masquer son propre statut de daigou. Comme évoqué plus haut, certaines marques identifient les daigous parmi leur clientèle et ces derniers se disent "parfois maltraités » en boutique. Venir accompagné (par un ami, un conjoint ou tout autre proche) permet d'atténuer les suspicions des vendeurs et de prendre des photos lors des essayages. Certaines personnes se font également payer pour faire la queue à la place des daigous : les consommateurs attendent parfois plus de quarante-cinq minutes avant d'entrer dans une boutique de marque. Pour dix-quinze euros, certains daigous font appel aux Chinois (souvent issus de milieux populaires) qui attendent pour eux. Citons également les cuisinières de nuit employées par des daigous en colocation qui, en raison du décalage horaire entre la France et la Chine, travaillent souvent sur un rythme décalé ; les transporteurs qui assurent, de manière personnalisée, le passage des marchandises aux frontières (la plupart du temps pour les produits de luxe, pour des raisons de temps et de sûreté). La rémunération de cette personne qui voyage avec le produit dans ses bagages peut atteindre $3 \%$ du prix de la transaction.

\section{La filière daigou comme catalyseur du renouvellement des activités des com- merçants wenzhous}

En sus des transporteurs qui collaborent avec les daigous à titre individuel et commande par commande (en fonction de qui rentre en Chine, où, à quel moment... et du produit transporté), de nombreuses entreprises de logistique, spécialisées dans les transports volumineux de marchandises, sont créées par des commerçants dits «classiques ", majoritairement originaires du Wenzhou. Elles proposent aux daigous un regroupement des produits à envoyer, sachant qu'un dédouanement ou une réduction des droits de douane par conteneur est possible. Ces entreprises de logistique, comme celles spécialisées dans la création de plateformes d'e-commerce (du même type qu'Alibaba et Jingdong 
Mall) visant à mettre directement en lien les consommateurs chinois et les fabricants français, représentent les deux principales voies du renouvellement des activités économiques transfrontalières, traditionnellement occupées par les migrants wenzhous. Ces commerçants sont souvent jeunes, issus de la migration chinoise 33 et savent mettre à profit leurs ressources distinctives (ethniques, linguistiques et sociales) en France et en Chine. Les collaborations avec les daigous font partie de leur stratégie d'exploitation du marché chinois. Comme le souligne Damien, 34 ans, originaire du Wenzhou et né à Paris :

«Sur notre plateforme d'e-commerce, nous commercialisons une trentaine de marques françaises, allant des couches de bébé aux vins. Ce sont des PME françaises, avec qui nous sommes en contrat de vente directe. Certaines marques ne sont pas encore connues en Chine. Mais je crois à l'avenir de ces petites marques sur le marché chinois et notre boulot, c'est justement de les aider à se faire connâ̂tre ! [Rires] Les daigous peuvent en partie nous aider à faire le marketing. Certains daigous sont contents de collaborer avec nous, notamment quand ils ont besoin d'un boulot stable. » (Entretien mené à Pairs en juillet 2015)

Ce que Damien appelle « collaboration » renvoie davantage à une relation d'employeur-employé. Les daigous perdent dans ce cas l'autonomie de leur activité et deviennent en quelque sorte les «commerciaux » des marques ecommercialisées par les commerçants wenzhous. Certains daigous en transition des études au travail choisissent en effet cette voie par souci de leur statut administratif en France (pour pouvoir continuer à renouveler leur titre de séjour).

\section{Alliés pour la contrefaçon: daigous et entrepreneurs de manufactures}

Tous les commerçants wenzhous ne renouvellent pas leurs activités économiques. Implantés de longue date dans les manufactures en France, certains continuent à fabriquer du prêt-à-porter, de la maroquinerie, de la quincaillerie, des produits ménagers..., tandis que les daigous, notamment ceux spécialisés dans les produits de luxe, cherchent à fournir des marchandises, face à la limitation par les marques de certains modèles et à la concurrence intraprofessionnelle entre daigous. Dans ce contexte, la commercialisation de contrefaçons sur le marché chinois parâtt « inévitable » aux yeux de nos enquêtés et

33. Ce constat rejoint les observations en Italie et en France sur la transformation des petites entreprises familiales chinoises en entreprises épousant les codes d'une économie transnationale et mondialisée, voir Merchionne G., Liu H., «Arts, cuisine et design. La génération 1.75 de la diaspora chinoise à Milan et la création d'entreprises transnationales ", Hommes $\mathcal{E}$ Migrations, 2016/2, n 1314, pp. 45-51 ; Chuang Y.-H., " Grandir dans une communauté commerçante. Les choix professionnels de jeunes issus de familles d'entrepreneurs wenzhou ", Hommes \& Migrations, 2016/2, n 1314, pp. 53-61. 
certains estiment que $10 \%$ des produits de luxe e-commercialisés via les daigous de la France vers la Chine relèvent de la contrefaçon.

Luchen, 29 ans, ancienne daigou dans la commercialisation de sacs de luxe, nous raconte en détail les activités d'une de ses homologues, connue pour trafic de faux sacs.

«Elle a été contactée par un commercial d'une usine de maroquinerie, en proche banlieue de Paris. Elle prend une commande et en informe l'usine. Ensuite, elle mobilise ses réseaux pour récupérer un emballage authentique de ce modèle de sac en question, souvent auprès de touristes chinois venus à Paris. Lorsque le sac est prêt, elle envoie séparément le sac et l'emballage à ses contacts en Chine, qui assembleront le tout. À un moment donné, elle est venue me voir en me proposant de travailler avec elle. Mais j'ai refusé [...] 》 (Entretien mené à Paris en octobre 2016).

La coopération entre des entrepreneurs wenzhous et des daigous fonctionne sur le partage d'un intérêt économique autour de la commercialisation des produits étrangers : les premiers assurent la fabrique et les seconds la vente grâce à leur réseau de clientèle développé. Selon Jing Daily ${ }^{34}$, il existe même des entreprises de contrefaçon localisées en Chine qui collaborent avec des daigous afin de déguiser leurs produits imités "made in China en produits fabriqués à l'étranger, grâce à l'ajout de détails comme des étiquettes d'expédition étrangères ou de faux reçus. Ces activités relevant de l'économie informelle et de circuits de contrefaçon internationaux sont difficiles à réguler, notamment à cause de la difficulté à collecter des preuves et de la présence de réseaux criminels aux frontières.

\section{Les velléités de contrôle de l'État sur les activités des daigous : une injonc- tion de mondialisation par le haut ?}

Puisque les activités de daigou entraînent un manque à gagner pour l'État chinois en raison du contournement des droits de douane et qu'elles peuvent alimenter d'autres formes d'économie informelle et de pratiques illégales, les autorités chinoises se sont engagées à contrôler cette pratique commerciale. Pour ce faire, plusieurs mesures ont été prises et mises en œuvre, dont en premier lieu des réformes fiscales visant à restreindre les marges commerciales des daigous.

34. Sim S., "China's Sketchy 'Daigou' Luxury Market Is A Hotbed For Fakes », Jing Dayily, 8 avril 2014 (https://jingdaily.com/chinas-sketchy-daigou-luxury-market-is-a-hotbed-forfakes/). 
Le ministère des Finances a tout d'abord engagé une réforme des taxes d'importations. Les taxes sur les vêtements qui oscillaient entre 14 et $23 \%$ sont désormais entre 7 et $10 \%$ et les taxes sur les chaussures sont réduites de 22-24\% à $12 \%$. Ensuite, comme nous l'avons déjà indiqué, le Conseil des affaires d'État a ajusté en avril 2016 le taux des taxes pour les « objets » et « marchandises ». Le seuil de transaction à usage personnel est fixé à 20000 $\mathrm{RMB}$ par personne et par an. Les taux de taxe pour les « objets » augmentent alors que ceux des «marchandises » sont en baisse. Certains produits - notamment des biens de consommations quotidiennes - sont exemptés de droits douaniers.

Convaincu que l'e-commercialisation représente l'avenir des échanges économiques, le gouvernement chinois favorise d'autres formes d'activités économiques transfrontalières que celle des daigous, notamment l'e-commerce via des plateformes mettant en contact direct les producteurs étrangers et les consommateurs chinois ${ }^{35}$. Treize villes chinoises ont été choisies en 2015 comme terrain pilote pour tester la combinaison de l'e-commerce transfrontalier et la vente au détail. L'État chinois a par ailleurs mis en ouvre des politiques d'accompagnement pour favoriser la création de plateformes d'etransactions. Par exemple, tous les Chinois diplômés et qualifiés à l'étranger dont font partie les daigous ici étudiés - peuvent bénéficier d'aides financières pour la création d'entreprise et d'exonération d'impôts pendant les premières années ${ }^{36}$. La nationalité chinoise ainsi que les titres scolaires sont ici valorisés comme ressources distinctives permettant de bénéficier des politiques étatiques d'investissement et de commerce, marquant une mondialisation par le haut.

Dans le cadre de collaborations bilatérales franco-chinoises, de multiples transactions de biens économiques et financiers entre les vendeurs/actionnaires français et les acquéreurs chinois ${ }^{37}$ se sont vues agrémentées de rencontres dédiées à l'e-commerce, avec la participation de chefs d'entreprises localisés en Chine et spécialisés dans l'e-transaction, de commerçants chinois en France majoritairement originaires du Wenzhou, et d'entrepreneurs français intéressés par l'e-export en Chine ${ }^{38}$. Ces rencontres ont permis la signature de plusieurs contrats de partenariat, parmi lesquels, en 2016, un contrat d'ap-

35. Le ministère du Commerce prévoit que les transactions via ce modèle commercial atteindront mille milliards de RMB en 2018.

36. Les clauses des politiques d'accompagnement diffèrent en fonction des caractéristiques des entreprises créées et des parcours antérieurs de leurs fondateurs - diplômés de telle ou telle université chinoise et/ou étrangère, adhérents au PCC ou non, originaires de telle région et de telle ethnie, etc.

37. Les filières sont variées et peuvent aller du secteur du vin aux centres de loisirs (hippodromes et golfs), en passant par les industries lourdes, la construction et la gestion commerciale, comme l'indique la consultation des actualités et informations publiées sur le site du « Bureau du Conseiller Économique et commercial de l'Ambassade de la République Populaire de Chine en République française » (中华人民共和国驻法兰西共和国大使馆经济商务参赞处) http://fr.mofcom.gov.cn/ 
provisionnement sur dix ans entre les entreprises chinoise Synutra et française Sodiaal pour la production de lait en poudre sur le territoire français à destination du marché chinois ${ }^{39}$. En permettant à l'entreprise chinoise située en France d'assurer directement la vente du produit en Chine, il s'agit de réduire la quantité de lait en poudre produite à l'étranger puis vendue en Chine via les daigous.

Face à l'émergence d'activités daigous, représentatives d'une mondialisation par le bas, plusieurs actions de l'État chinois et d'entreprises multinationales sont entreprises pour favoriser la libre circulation des marchandises et des capitaux, et donc une mondialisation par le haut. Ces deux voies de mondialisation sont ainsi imbriquées et s'influencent mutuellement. Quant à l'État français, nous constatons une régulation formelle relativement faible de l'économie informelle développée au sein de la population chinoise en France (contrefaçon, fraude fiscale).

Le mode d'achat daigou est exemplaire d'une économie transnationale par le bas. Il est structuré par des flux de biens, d'informations, de personnes et de capitaux entre différents marchés qui fonctionnent comme des nœuds dans un système mondial non hégémonique ${ }^{40}$.

À partir de l'étude des pratiques intraprofessionnelles mais également interprofessionnelles de la filière daigou, nous avons montré la capacité des agents daigou à " être ici et là-bas en même temps 41 ", la reconfiguration des réseaux commerciaux transnationaux (incluant les réseaux souterrains $\mathrm{du}$ commerce informel) à l'ère numérique d'une société chinoise de consommation, les trajectoires et logiques migratoires différenciées et hiérarchisées au sein des daigous, et le rôle moteur joué par ces derniers dans le processus de mondialisation avant même la mise en œuvre de politiques de régulation et de dispositifs de contrôle. À ce titre, étudier les activités et les pratiques de ces acteurs ordinaires offre un autre regard sur l'international, complémentaire à ce qui peut être saisi « par le haut ", à savoir l'analyse des actions des États et des multinationales.

38. Pour plus de détails, voir entre autres le compte-rendu du China Development Forum qui a eu lieu le 3 juin 2015.

39. La coopérative devra collecter chaque année auprès de 800 producteurs de la région bretonne environ 300 millions de litres de lait. L'objectif pour le groupe chinois est de produire 100000 tonnes de poudre de lait par an à destination du marché chinois (http://fr.mofcom.gov.cn/article/zxhz/201707/20170702615331.shtml).

40. Mathews G., Ribeiro G. L. et Vega C. A. (eds.), Globalization from Below: The World's Other Economy, Londres, Routledge, 2012.

41. Santelli E., «Entre ici et là-bas : les parcours d'entrepreneurs transnationaux. Investissement économique en Algérie des descendants de l'immigration algérienne de France », Sociologie, vol. 1, n 3, 2010, pp. 393-411. 
En termes de "propriétés sociales », les daigous étudiés ici, tous qualifiés, ne sont pas issus des classes populaires, acteurs usuels de la mondialisation par le bas ${ }^{42}$. De par sa nouveauté, son ampleur, l'intensité des capitaux véhiculés, ainsi que la complexité des réseaux économiques qu'elle occasionne, l'activité des daigous mérite d'être étudiée. L'analyse de cette filière économique nous semble particulièrement féconde pour interroger les nouveaux sens à donner aux acteurs de la mondialisation par le bas (lorsque ces derniers sont qualifiés), ainsi que pour enquêter sur les caractéristiques spécifiques de la société chinoise de consommation (manque de confiance envers les produits locaux, ethos consommateur d'une Chine en moyennisation, etc.).

Par ailleurs, notre étude montre que les plus anciens migrants chinois en France (les Wenzhous) et les plus récents (qui sont aussi les plus qualifiés et dont sont issus les daigous) s'engagent de manières différentes dans des actions transnationales, ici en l'occurrence l'e-commerce. Parmi les daigous, les plus dotés en ressources sociales et culturelles font circuler dans la société d'accueil à la fois des produits et des capitaux, des styles de vie, des services et des connaissances.

Enfin, la notion du «temps » s'avère fructueuse dans l'analyse des phénomènes de mondialisation. Elle permet d'articuler des échelles analytiques micro, méso et macro, par l'analyse des parcours des acteurs économiques à travers une approche de trajectoire sociale ; par l'étude des relations interprofessionnelles au sein de l'immigration chinoise en tenant compte de son histoire ; et enfin par l'investigation historicisée de l'évolution des formes d'activités économiques entre la France et la Chine ainsi que de ses contextes socio-économico-politiques. Une autre réflexion méthodologique que nous menons concerne l'apport des approches « qualitatives » et « multisituées » 43 dans les études des activités économiques transnationales, largement démontré par des chercheurs en études migratoires ${ }^{44}$. Sur notre terrain, l'économie et les réseaux informels investis par les daigous ne peuvent être saisis que de près, à condition d'établir une relation de confiance entre enquêteur et enquêtés.

42. Tarrius A., op. cit.

43. Sur l'approche " ethnographie multi-située », voir Marcus G. E., «Ethnography in/of the World System: The Emergence of multi-sited Ethnography ", Annual Review of Anthropology, vol. 24, 1995, pp. 95-117.

44. Voir entre autres Tarrius A., op. cit.; Peraldi M. (dir.), La fin des norias ? Réseaux migrants dans les économies marchandes en Méditerranée, Paris, Maisonneuve\&Larose, 2002 ; Berthomière W., Hily M.-A., « Décrire les migrations internationales. Les expériences de la co-présence ", Revue européenne des migrations internationales, vol. 22, n ${ }^{\circ}$, 2006, pp. 67 82 . 